\title{
Surface-Integral Approach to the Coulomb Few-Body Scattering Problem
}

\author{
A. S. Kadyrovi,a , I. Bray ${ }^{1}$, A. M. Mukhamedzhanov' ${ }^{2}$, and A. T. Stelbovics ${ }^{1}$ \\ 1 Curtin University, GPO Box U1987, Perth, WA 6845, Australia \\ 2 Cyclotron Institute, Texas A\&M University, College Station, Texas 77843, USA
}

\begin{abstract}
We present main features of a surface-integral approach to the Coulomb few-body scattering problem. This approach is valid for both short-range and Coulombic longe-range interactions. We give new general definitions for the potential scattering amplitude. For the Coulombic potentials the generalized amplitude gives the physical on-shell amplitude without recourse to a renormalization procedure. New post and prior forms for the amplitudes of breakup, direct and rearrangement scattering in a Coulomb three-body system are also presented. The Green's functions and formal solutions of the Schrödinger equation in integral form are not used. Therefore, for the purpose of defining the scattering amplitudes the knowledge of a complicated analytic structure of the Green's function in the complex-energy plane is not required.
\end{abstract}

\section{Introduction}

Atomic and subatomic many-particle systems with the long-range Coulomb interactions and cosmological many-body systems with the gravitational interactions are of fundamental importance for our understanding of the universe. Atomic and nuclear systems are governed by the pairwise Coulomb potential (in addition to other possible short-range interactions) which is proportional to the charges of interacting particles and inversely proportional to the interparticle distance $(r)$. In case of cosmic objects the masses of the objects play the role of gravitational charges. This $1 / r$ potential is arguably the most important interaction in the structure of the universe. The long-range character of this potential implies that there is interaction even at very large (in fact, infinitely large) distances. This leads to various divergency problems in many branches of physics from quantum mechanics to quantum electrodynamics, from field theories to statistical physics, from celestial mechanics to cosmology. Despite being identified from the very beginning of modern physics, these problems have resisted proper solution. As a result different renormalization theories have been developed aimed at their ad hoc resolution. However, such approaching have been possible only in some limited class of problems. One can think that a simple cut off beyond some finite range could be a remedy for these problems. Unfortunately, this is not the case for most systems of practical interest. This could be because limiting the range of the potential leads to the loss of information about the very nature of the field creating the potential that cannot be recovered by simply taking the screening range to infinity.

\footnotetext{
${ }^{a}$ e-mail: a.kadyrov@curtin.edu.au
}

It is well known that conventional quantummechanical collision theory is valid only when particles interact via short-range potentials. For charged particles the theory requires modification due to the fact that the long range of the Coulomb potential distorts the incident and scattered waves right out to infinity. Formal scattering theory is generalized to include Coulomb long-range potentials using renormalization methods [1]. The renormalization theories lead to the correct cross sections for the two-body problem, however, the results from these procedures cannot be regarded as completely consistent. For instance, in screening-based renormalization methods different ways of screening lead to different asymptotic forms for the scattering wave function. These asymptotic forms differ from the exact one obtained from the solution of the Schrödinger equation. Moreover the renormalization methods give rise to a scattering amplitude that does not exist on the energy shell. In other words, the resulting amplitude cannot be directly used for calculating cross sections. This is because the amplitude obtained in these methods has complex factors which are divergent on the energy shell. Therefore, these factors must be removed (renormalized) before approaching the on-shell point. Furthermore, the renormalization factors depend on the way the limits are taken when the on-shell point is approached. In other words, depending on the way you take the limits different factors need to be removed. Thus, the ad-hoc renormalization procedure is not logically consistent. In quantum collision theory it is customary to define the scattering amplitude in terms of the scattering wavefunction and the potential of interaction. Despite the fact that the Coulomb wavefunction and the Coulomb potential are both known analytically, the conventional theory has not been able to provide such 
a standard definition for the amplitude of scattering of two charged particles, which yields the Rutherford cross section.

The situation with the Coulomb few-body scattering problem is significantly more complicated. Rigorous scattering theory for a system of three particles valid for short-range potentials was given by Faddeev $[2,3]$. For the charged particles with the longrange Coulomb interaction the theory has faced difficulties associated with the compactness of the underlying equations. A renormalization method was implemented successfully for the three-body problem when only two particles are charged [4]. There are no compact integral equations yet known for collisions of more than two charged particles that are satisfactory above the breakup threshold [1]. Furthermore, there is no theoretical proof or practical evidence that a renormalization approach can be applied to the Faddeev equations for the three-body Coulomb problem. This has serious consequences especially for threebody problems in atomic physics where all three particles are charged.

As far as breakup of a bound state of two particles in a system of three charged particles is concerned, the key problem is how to extract the scattering information from the wavefunction when the latter is available. The conventional theory fails to provide a formal post-form definition of the breakup amplitude for three charged particles in terms of the total wavefunction with outgoing scattered waves describing the process. Therefore, the Coulomb interaction is screened and the formula for the short-range case is used. However, it is well known that the short-range definition of the breakup amplitude diverges when the screening radius is taken to infinity. Thus we have a situation where we cannot use the theory unless we screen the Coulomb interaction, and when we do, we end up with quantities which diverge as screening is removed. This leaves no choice but to use renormalization to fix unphysical results. As mentioned above this is not possible in all cases of interest. Therefore, a new approach to the Coulomb three-body problem that does not need renormalization is required.

Despite the aforementioned problems remarkable progress has been achieved in atomic physics in describing (e,2e) processes via the exterior complexscaling (ECS) $[5,6]$ and the convergent close-coupling (CCC) [7] methods. In particular, the success of the ECS approach to Coulomb breakup caused us to reexamine the underlying formal theory $[8,9]$. In the ECS method the amplitude is calculated from Peterkop's trial integral [10] which is likely to be some kind of approximation to the exact breakup amplitude in the post form (unavailable in conventional scattering theory). In the CCC method one of the electrons is treated using a square-integrable $\left(L^{2}\right)$ representation, and the breakup amplitude can be related to a particular form of Peterkop's integral. Despite the success of the computational methods in describing the measured cross sections, the traditional formal the- ory of scattering was unable to provide a definition for the breakup amplitude in terms of the scattered wave. This has been a long-standing problem [11].

In the present work we outline a surface-integral approach to formulating scattering theory. This represents an extension of the general formalism of scattering theory to systems of two and three charged particles with long-range Coulombic interactions. This paper is addressed to readers who are not interested in mathematical detail. However, further details of the present formalism can be found in Ref. [12].

\section{Two-body scattering problem}

We first present an alternative formulation of potential scattering theory. Essential feature of this formulation is that it avoids the reference to the Green's function and formal solution for the scattered wavefunction in the integral form. This leads to new more general definitions for scattering amplitude valid for arbitrary interactions including Coulombic long-range ones.

Wave functions we deal with in scattering theory go beyond the Hilbert space. Since these functions are not square-integrable $\left(L^{2}\right)$ their scalar products can be unbounded. While this fact is not a problem on its own, nevertheless, non- $L^{2}$ functions do make certain integrals emerging in the theory divergent. In case of integrals containing the interaction potential a standard procedure, which ensures their existence, is limiting the range of the potential. However, this irreversibly distorts the nature of the problem. Throughout this work we use a different approach to dealing with the aforementioned problem. We first formulate the scattering problem in a finite region of coordinate space and then extend it to the full space.

\subsection{Scattering via short-range interaction}

Let us consider a system of two interacting particles via a real central potential $V(r)$. The scattering state of this system is a solution to the Schrödinger equation (SE)

$$
(\epsilon-h) \psi_{\mathbf{k}}^{ \pm}(\mathbf{r})=0
$$

where $h=h_{0}+V$ is the total two-body Hamiltonian of the system, $h_{0}=-\Delta_{\mathbf{r}} / 2 \mu$ is the free Hamiltonian operator, $\epsilon=k^{2} / 2 \mu$ is the energy of the relative motion, $\mathbf{r}$ is the relative coordinate of the particles 1 and 2 and $\mathbf{k}$ is their relative momentum, $\mu$ is the reduced mass. We begin by supposing that the interaction potential $V$ is short-ranged. Traditionally, we would introduce here some definition of the short-rangeness. Such a definition would involve convergence of a certain integral containing the wavefunctions $\psi_{\mathbf{k}}^{ \pm}$and the potential $V$. Our approach is based on partial inner 
products which we define according to

$$
\left\langle f_{\mathbf{k}^{\prime}} \mid g_{\mathbf{k}}\right\rangle_{r_{0}}=\int_{r \leq r_{0}} d \mathbf{r} f_{\mathbf{k}^{\prime}}^{*}(\mathbf{r}) g_{\mathbf{k}}(\mathbf{r})
$$

where the integration is limited to the volume of a sphere of radius $r_{0}$. Generally speaking, the full scalar product $\left\langle f_{\mathbf{k}^{\prime}} \mid g_{\mathbf{k}}\right\rangle$ can be unbounded. Thus our approach does not require the short-rangeness as far as integrals are concerned. All integrals converge just because we are in a limited space. Therefore, without going into details, we simply assume the following definition of the short-rangeness: $V$ is such that the asymptotic boundary condition for $\psi_{\mathbf{k}}^{+}$is given, in the leading order, as

$$
\psi_{\mathbf{k}}^{+}(\mathbf{r}) \stackrel{r \rightarrow \infty}{\sim} e^{i \mathbf{k} \cdot \mathbf{r}}+f(\widehat{\mathbf{k}} \cdot \widehat{\mathbf{r}}) \frac{e^{i k r}}{r} .
$$

The second suitable solution $\psi_{\mathbf{k}}^{-}(\mathbf{r})$ can be found from the well known relationship

$$
\psi_{\mathbf{k}}^{-}(\mathbf{r})=\left[\psi_{-\mathbf{k}}^{+}(\mathbf{r})\right]^{*} .
$$

We can separate $\psi_{\mathbf{k}}^{ \pm}$into the incident and the scattered parts according to

$$
\psi_{\mathbf{k}}^{ \pm}(\mathbf{r})=\phi_{\mathbf{k}}(\mathbf{r})+\psi_{\mathbf{k}}^{\mathrm{sc} \pm}(\mathbf{r}),
$$

where $\phi_{\mathbf{k}}(\mathbf{r})=e^{i \mathbf{k} \cdot \mathbf{r}}$. Then Eq. (1) can be written in the form

$$
(\epsilon-h) \psi_{\mathbf{k}}^{\mathrm{sc} \pm}(\mathbf{r})=(h-\epsilon) \phi_{\mathbf{k}}(\mathbf{r}) .
$$

Let us multiply Eq. (6) for $\psi_{\mathbf{k}}^{\mathrm{sc}+}$ by $\psi_{\mathbf{k}^{\prime}}^{-*}(\mathbf{r})$ from the left and integrate the result over the volume of a sphere of radius $r_{0}$ :

$$
\left\langle\psi_{\mathbf{k}^{\prime}}^{-} \mid(\epsilon-h) \psi_{\mathbf{k}}^{\mathrm{sc}+}\right\rangle_{r_{0}}=\left\langle\psi_{\mathbf{k}^{\prime}}^{-} \mid(h-\epsilon) \phi_{\mathbf{k}}\right\rangle_{r_{0}},
$$

where $\psi_{\mathbf{k}^{\prime}}^{-}(\mathbf{r})$ is another solution of the SE at a different momentum but the same energy $\epsilon$, i.e. $k^{\prime}=k$. In other words, $\psi_{\mathbf{k}^{\prime}}^{-}(\mathbf{r})$ is the eigenfunction of the operator $h$, therefore we also can write

$$
\left\langle(\epsilon-h) \psi_{\mathbf{k}^{\prime}}^{-} \mid \psi_{\mathbf{k}}^{\mathrm{sc}+}\right\rangle_{r_{0}}=0 .
$$

Subtracting Eq. (8) from (7) we get

$$
\begin{array}{r}
\left\langle\psi_{\mathbf{k}^{\prime}}^{-} \mid(\epsilon-h) \psi_{\mathbf{k}}^{\mathrm{sc}+}\right\rangle_{r_{0}}-\left\langle(\epsilon-h) \psi_{\mathbf{k}^{\prime}}^{-} \mid \psi_{\mathbf{k}}^{\mathrm{sc}+}\right\rangle_{r_{0}}= \\
\left\langle\psi_{\mathbf{k}^{\prime}}^{-} \mid(h-\epsilon) \phi_{\mathbf{k}}\right\rangle_{r_{0}} .
\end{array}
$$

Canceling the terms containing $\epsilon-V$ (these terms are finite because we are in a limited space) and letting $r_{0} \rightarrow \infty$ on both sides of the equation we have

$$
\begin{array}{r}
\lim _{r_{0} \rightarrow \infty}\left[-\left\langle\psi_{\mathbf{k}^{\prime}}^{-} \mid h_{0} \psi_{\mathbf{k}}^{\mathrm{sc}+}\right\rangle+\left\langle h_{0} \psi_{\mathbf{k}^{\prime}}^{-} \mid \psi_{\mathbf{k}}^{\mathrm{sc}+}\right\rangle\right]_{r_{0}}= \\
\lim _{r_{0} \rightarrow \infty}\left\langle\psi_{\mathbf{k}^{\prime}}^{-} \mid(h-\epsilon) \phi_{\mathbf{k}}\right\rangle_{r_{0}}
\end{array}
$$

Note that the left hand side of Eq. (10) would vanish if the operator $h_{0}$ were Hermitian. Now we calculate both sides of the equation independently. First we investigate the limit on the left-hand side (LHS) which can be written as

$$
\begin{aligned}
\text { LHS }= & \lim _{r_{0} \rightarrow \infty}\left[-\frac{1}{2 \mu} r^{2} \int d \hat{\mathbf{r}}\right. \\
& \left.\times\left(\psi_{\mathbf{k}}^{\mathrm{sc}+} \frac{\partial \psi_{\mathbf{k}^{\prime}}^{-*}}{\partial r}-\psi_{\mathbf{k}^{\prime}}^{-*} \frac{\partial \psi_{\mathbf{k}}^{\mathrm{sc}+}}{\partial r}\right)\right]_{r=r_{0}} .
\end{aligned}
$$

Above we used the Green's theorem to transform the volume integral into the surface integral. Using the asymptotic form of the plane wave (see, e.g., [13])

$$
e^{i \mathbf{k} \cdot \mathbf{r} r} \stackrel{\sim}{\sim} \frac{2 \pi}{i k r}\left[e^{i k r} \delta(\widehat{\mathbf{k}}-\widehat{\mathbf{r}})-e^{-i k r} \delta(\widehat{\mathbf{k}}+\widehat{\mathbf{r}})\right],
$$

we get from Eq. (11)

$$
\text { LHS }=-\frac{2 \pi}{\mu} f\left(\hat{\mathbf{k}}^{\prime} \cdot \hat{\mathbf{k}}\right) .
$$

Since $-2 \pi / \mu f=t$ we can write Eq. (10) as

$$
t\left(\mathbf{k}^{\prime}, \mathbf{k}\right)=\lim _{r_{0} \rightarrow \infty}\left\langle\psi_{\mathbf{k}^{\prime}}^{-} \mid(h-\epsilon) \phi_{\mathbf{k}}\right\rangle_{r_{0}} .
$$

Thus we have got a definition for the on-shell transition matrix. We emphasize that, this definition has emerged as a result of a surface integral which has not vanished. In addition, in order to show this there was no need to use a formal solution of the SE in the integral form in contrast to [14].

We now consider Eq. (6) for $\psi_{\mathbf{k}^{\prime}}^{\mathrm{sc}-}$ and multiply it by $\psi_{\mathbf{k}}^{+}$from the right. Integrating the result over the volume of a sphere of radius $r_{0}$ we have

$$
\left\langle(\epsilon-h) \psi_{\mathbf{k}^{\prime}}^{\mathrm{sc}-} \mid \psi_{\mathbf{k}}^{+}\right\rangle_{r_{0}}=\left\langle(h-\epsilon) \phi_{\mathbf{k}^{\prime}} \mid \psi_{\mathbf{k}}^{+}\right\rangle_{r_{0}},
$$

where again $k^{\prime}=k$. We also consider

$$
\left\langle\psi_{\mathbf{k}^{\prime}}^{\mathrm{sc}-} \mid(\epsilon-h) \psi_{\mathbf{k}}^{+}\right\rangle_{r_{0}}=0,
$$

which is valid for arbitrary $r_{0}$ due to Eq. (1). Subtracting Eq. (16) from (15) we get

$$
\begin{array}{r}
\left\langle(\epsilon-h) \psi_{\mathbf{k}^{\prime}}^{\mathrm{sc}-} \mid \psi_{\mathbf{k}}^{+}\right\rangle_{r_{0}}-\left\langle\psi_{\mathbf{k}^{\prime}}^{\mathrm{sc}-} \mid(\epsilon-h) \psi_{\mathbf{k}}^{+}\right\rangle_{r_{0}}= \\
\left\langle(h-\epsilon) \phi_{\mathbf{k}^{\prime}} \mid \psi_{\mathbf{k}}^{+}\right\rangle_{r_{0}} .
\end{array}
$$

This equation is similar to Eq. (9) in form. Taking $r_{0} \rightarrow \infty$ limit on both sides and calculating the LHS in a similar way we find the second form for the scattering amplitude

$$
t\left(\mathbf{k}^{\prime}, \mathbf{k}\right)=\lim _{r_{0} \rightarrow \infty}\left\langle(h-\epsilon) \phi_{\mathbf{k}^{\prime}} \mid \psi_{\mathbf{k}}^{+}\right\rangle_{r_{0}} .
$$

We call Eqs. (14) and (18) the prior (pr) and post (pt) form definitions of the scattering amplitude, respectively.

Eqs. (14) and (18) immediately suggest that the scattering amplitude can take surface-integral forms. Indeed, since

$$
\left\langle(\epsilon-h) \psi_{\mathbf{k}^{\prime}}^{-} \mid \phi_{\mathbf{k}}\right\rangle_{r_{0}}=0
$$


from Eq. (14) we have

$$
t^{\mathrm{pr}}\left(\mathbf{k}^{\prime}, \mathbf{k}\right)=\lim _{r_{0} \rightarrow \infty}\left[\left\langle\psi_{\mathbf{k}^{\prime}}^{-} \mid h_{0} \phi_{\mathbf{k}}\right\rangle-\left\langle h_{0} \psi_{\mathbf{k}^{\prime}}^{-} \mid \phi_{\mathbf{k}}\right\rangle\right]_{r_{0}},
$$

which can be written as

$$
\begin{aligned}
t^{\mathrm{pr}}\left(\mathbf{k}^{\prime}, \mathbf{k}\right)= & -\frac{1}{2 \mu} \lim _{r_{0} \rightarrow \infty} r_{0}^{2} \int d \hat{\mathbf{r}} \\
& \times\left[\psi_{\mathbf{k}^{\prime}}^{-*} \frac{\partial \phi_{\mathbf{k}}}{\partial r}-\phi_{\mathbf{k}} \frac{\partial \psi_{\mathbf{k}^{\prime}}^{-*}}{\partial r}\right]_{r_{0}} .
\end{aligned}
$$

At the same time using the fact that

$$
\left\langle\phi_{\mathbf{k}^{\prime}} \mid(h-\epsilon) \psi_{\mathbf{k}}^{+}\right\rangle_{r_{0}}=0,
$$

from Eq. (18) we also get

$$
t^{\mathrm{pt}}\left(\mathbf{k}^{\prime}, \mathbf{k}\right)=\lim _{r_{0} \rightarrow \infty}\left[\left\langle h_{0} \phi_{\mathbf{k}^{\prime}} \mid \psi_{\mathbf{k}}^{+}\right\rangle-\left\langle\phi_{\mathbf{k}^{\prime}} \mid h_{0} \psi_{\mathbf{k}}^{+}\right\rangle\right]_{r_{0}},
$$

which transforms to

$$
\begin{aligned}
t^{\mathrm{pt}}\left(\mathbf{k}^{\prime}, \mathbf{k}\right)= & -\frac{1}{2 \mu} \lim _{r_{0} \rightarrow \infty} r_{0}^{2} \int d \hat{\mathbf{r}} \\
& \times\left[\psi_{\mathbf{k}}^{+} \frac{\partial \phi_{\mathbf{k}^{\prime}}^{*}}{\partial r}-\phi_{\mathbf{k}^{\prime}}^{*} \frac{\partial \psi_{\mathbf{k}}^{+}}{\partial r}\right]_{r_{0}} .
\end{aligned}
$$

Thus the scattering T-matrix, conventionally given as a volume integral, can be written equivalently in surface-integral forms. We emphasize that in these forms the T-matrix depends only on the asymptotic behavior of the participating functions. Therefore, generally speaking, knowledge of the scattering wavefunction in the internal (nonasymptotic) region is not required. In addition, the surface-integral forms are readily expanded in partial waves leading to a simple result containing only the limiting procedure. Therefore, these forms are particularly suitable for practical calculations.

The same analysis as used above to calculate the LHS of Eq. (10) can be performed on the surfaceintegral representations $(21)$ and $(24)$ to yield $t\left(\mathbf{k}^{\prime}, \mathbf{k}\right)$. This means that the limits on the RHS of Eqs. (14) and (18) also exist. Therefore from Eqs. (14) and (18) we get the general volume-integral prior and post forms of the scattering amplitude

$$
\begin{aligned}
& t^{\mathrm{pr}}\left(\mathbf{k}^{\prime}, \mathbf{k}\right)=\left\langle\psi_{\mathbf{k}^{\prime}}^{-} \mid(h-\epsilon) \phi_{\mathbf{k}}\right\rangle, \\
& t^{\mathrm{pt}}\left(\mathbf{k}^{\prime}, \mathbf{k}\right)=\left\langle(h-\epsilon) \phi_{\mathbf{k}^{\prime}} \mid \psi_{\mathbf{k}}^{+}\right\rangle .
\end{aligned}
$$

These forms are consistent with the conventional theory. In order to see this we note that

$$
(h-\epsilon) \phi_{\mathbf{k}}(\mathbf{r})=V \phi_{\mathbf{k}}(\mathbf{r}) .
$$

Therefore, the two forms given by Eqs. (14) and (18), respectively, are identical to the standard prior and post forms of the T-matrix

$$
\begin{aligned}
& t^{\mathrm{pr}}\left(\mathbf{k}^{\prime}, \mathbf{k}\right)=\left\langle\psi_{\mathbf{k}^{\prime}}^{-}|V| \phi_{\mathbf{k}}\right\rangle, \\
& t^{\mathrm{pt}}\left(\mathbf{k}^{\prime}, \mathbf{k}\right)=\left\langle\phi_{\mathbf{k}^{\prime}}|V| \psi_{\mathbf{k}}^{+}\right\rangle,
\end{aligned}
$$

usually obtained in literature from the formal solution of Eq. (1) using the asymptotic form of the free Green's function. We shall show in the following subsection that the T-matrices as written in Eqs. (14) and (18) are more general and valid for arbitrary interaction potentials provided the function on which the operator $(h-\epsilon)$ is applied is the leading-order incident wave.

\subsection{Scattering via Coulomb-like interaction}

We assume now that interaction $V$ consists of some short-range part and the Coulomb potential. A scattering state in a system of two charged particles is still the solution to the Schrödinger equation (1). However, when the potential has the Coulomb tail the scattering wavefunction $\psi_{\mathbf{k}}^{+}(\mathbf{r})$ asymptotically behaves like the Coulomb-modified plane wave and a Coulombmodified outgoing spherical wave

$$
\begin{aligned}
\psi_{\mathbf{k}}^{+}(\mathbf{r}) \stackrel{r \rightarrow \infty}{\sim} e^{i \mathbf{k} \cdot \mathbf{r}+i \gamma \ln (k r-\mathbf{k} \cdot \mathbf{r})} \\
+f(\widehat{\mathbf{k}} \cdot \widehat{\mathbf{r}}) \frac{e^{i k r-i \gamma \ln (2 k r)}}{r},
\end{aligned}
$$

where $\gamma$ is the Sommerfeld parameter. The second suitable solution $\psi_{\mathbf{k}}^{-}(\mathbf{r})$ and its asymptotic behavior are obtained using Eq. (4).

As the form of Eq. (30) suggests, the leading order terms in the asymptotic region already contain all the scattering information we want. The next order terms simply repeat this information. Therefore, all we need for extracting the scattering amplitude is the leading-order asymptotic term of the scattered wave $\psi_{\mathbf{k}}^{\mathrm{sc} \pm}$. Therefore, let us denote the leading-order incident waves as

$$
\phi_{\mathbf{k}}^{(0) \pm}(\mathbf{r})=e^{i \mathbf{k} \cdot \mathbf{r} \pm i \gamma \ln (k r \mp \mathbf{k} \cdot \mathbf{r})},
$$

and single them out in $\psi_{\mathbf{k}}^{ \pm}$according to

$$
\psi_{\mathbf{k}}^{ \pm}(\mathbf{r})=\phi_{\mathbf{k}}^{(0) \pm}(\mathbf{r})+\psi_{\mathbf{k}}^{\mathrm{sc} \pm}(\mathbf{r})
$$

The splitting according to Eq. (32) represents the logical fact that the unscattered incident wave is coming from infinity and should be taken in a form valid at asymptotically large distances (see [12] for more discussion).

With these prerequisites repeating the algebra of the previous subsection we get

$$
\begin{aligned}
& t^{\mathrm{pr}}\left(\mathbf{k}^{\prime}, \mathbf{k}\right)=\lim _{r_{0} \rightarrow \infty}\left\langle\psi_{\mathbf{k}^{\prime}}^{-} \mid(h-\epsilon) \phi_{\mathbf{k}}^{(0)+}\right\rangle_{r_{0}}, \\
& t^{\mathrm{pt}}\left(\mathbf{k}^{\prime}, \mathbf{k}\right)=\lim _{r_{0} \rightarrow \infty}\left\langle(h-\epsilon) \phi_{\mathbf{k}^{\prime}}^{(0)-} \mid \psi_{\mathbf{k}}^{+}\right\rangle_{r_{0}} .
\end{aligned}
$$

In leading to these results the two integrals containing $\epsilon-V$ cancel each other as in Eqs. (9) and (10). One can argue that such integrals diverge unless $V$ is short ranged. This is true but only for the whole space. The canceled integrals are over the limited space and are 
$19^{\text {th }}$ International IUPAP Conference on Few-Body Problems in Physics

finite. This emphasizes the whole idea behind working in a limited space which is to make potentially divergent terms disappear. Another difference is that while evaluating integrals similar to those we had in Eq. (11) we use an asymptotic form of the distorted plane waves $\phi_{\mathbf{k}}^{(0) \pm}(\mathbf{r})[14]$ :

$$
\begin{aligned}
e^{i \mathbf{k} \cdot \mathbf{r} \pm i \gamma \ln (k r \mp \mathbf{k} \cdot \mathbf{r}) \stackrel{r \rightarrow \infty}{\longrightarrow}} & \frac{2 \pi}{i k r}\left[e^{i k r-i \gamma \ln (2 k r)} \delta(\widehat{\mathbf{k}}-\widehat{\mathbf{r}})\right. \\
& \left.-e^{-i k r+i \gamma \ln (2 k r)} \delta(\widehat{\mathbf{k}}+\widehat{\mathbf{r}})\right]
\end{aligned}
$$

The physical meaning of this replacement is quite obvious: the distorted plane waves correspond to two different fluxes of particles; two particles approaching each other are represented by the incoming wave and particles going away from each other are described by the outgoing wave. In the absence of the long-range distortion Eq. (35) transforms to the familiar asymptotic form of the plane wave (Eq. (12)). The usage of the replacement (35) is discussed in [12].

It is not difficult to see that the results given above are consistent with conventional potential scattering theory for short-range interactions. The existing formulation of scattering theory relies on the condition that interaction $V(r)$ decreases faster than the Coulomb interaction when $r \rightarrow \infty[\gamma=0$ in Eq. (31)], so that

$$
\phi_{\mathbf{k}}^{(0) \pm}(\mathbf{r}) \rightarrow \phi_{\mathbf{k}}(\mathbf{r})=e^{i \mathbf{k} \cdot \mathbf{r}} .
$$

Therefore, our results transform to Eqs. (14) and (18) which, in the light of Eq. (27), further reduce to Eqs. (28) and (29) in agreement with the standard definitions of the T-matrix. Obviously, when interaction $V$ has a $1 / r$ tail, $\phi_{\mathbf{k}}^{(0)+}$ does not satisfy Eq. (27). As a result conventional definitions become invalid for Coulomb-like potentials. On the other hand, when the interaction is purely Coulomb, we can proceed further with analytical methods. In this case $\psi_{\mathbf{k}}^{ \pm}$are the well known Coulomb waves (see, e.g. Ref. [1]). Then the matrix elements in Eqs. (33) and (34) can be evaluated and shown to give the well-known on-shell Coulomb T-matrix [14].

\section{The Coulomb three-body scattering problem}

The same idea can be applied to a few-body scattering problem. Let us consider a system of three charged particles. We use the Jacobi coordinates where $\mathbf{r}_{\alpha}$ is the relative coordinate, and $\mathbf{k}_{\alpha}$ is the relative momentum, between particles $\beta$ and $\gamma, \boldsymbol{\rho}_{\alpha}$ is the relative coordinate of the center of mass of the pair $(\beta, \gamma)$ and particle $\alpha$, with $\mathbf{q}_{\alpha}$ being the canonically conjugate relative momentum. Accordingly, $\mu_{\alpha}$ is the reduced mass of particles $\beta$ and $\gamma$ and $M_{\alpha}$ is the reduced mass of the pair $(\beta, \gamma)$ and particle $\alpha$. Here $\alpha, \beta, \gamma=1,2,3$, and $\alpha \neq \beta \neq \gamma$.
We are interested in scattering of particle $\alpha$ with incident momentum $\mathbf{q}_{\alpha n}$ off a bound pair $(\beta, \gamma)$ in initial state $\phi_{\alpha n}\left(\mathbf{r}_{\alpha}\right)$ of energy $E_{\alpha n}$. Here $n$ denotes a full set of quantum numbers of the bound state $(\beta, \gamma)$ in channel $\alpha$. Assume that the energy of the projectile $q_{\alpha n}^{2} / 2 M_{\alpha}$ is enough to break up the target. Note that, in addition to direct scattering and rearrangement $(\beta+(\gamma, \alpha))$ channels, there is a breakup one. Therefore, we call this $2 \rightarrow 3$ process. In order to find the amplitudes of direct scattering, rearrangement and breakup in this collision we need the total scattering wavefunction developed from the initial channel $\alpha n$ and three different asymptotic wavefunctions corresponding to three final-state channels. The same amplitudes can be found in the so-called prior forms as well, which requires the knowledge of the other three types of the total scattering wavefunctions being developed to three different final-state wavefunctions. Thus, in any case, we require a set of four total scattering wavefunctions together with their corresponding asymptotic forms in all relevant asymptotic domains.

There are two distinct types of asymptotic domains. Let us call $\Omega_{0}$ the asymptotic domain, where all interparticle distances are large, i.e, $r_{\alpha} \rightarrow \infty$, $\rho_{\alpha} \rightarrow \infty$, so that $r_{\alpha} / \rho_{\alpha}$ is non-zero. In addition, we call $\Omega_{\alpha}$ the asymptotic regime, where $\rho_{\alpha} \rightarrow \infty$, however $r_{\alpha}$ satisfies the constraint $r_{\alpha} / \rho_{\alpha} \rightarrow 0$.

The total three-body wavefunction describing the $2 \rightarrow 3$ processes satisfies the Schrödinger equation

$$
(E-H) \Psi_{\alpha n}^{+}\left(\mathbf{r}_{\alpha}, \boldsymbol{\rho}_{\alpha}\right)=0,
$$

with outgoing-wave boundary conditions, where $H=$ $H_{0}+V, H_{0}=-\Delta_{\mathbf{r}_{\alpha}} / 2 \mu_{\alpha}-\Delta_{\boldsymbol{\rho}_{\alpha}} / 2 M_{\alpha}$ is the free Hamiltonian, $V=V_{\alpha}\left(\mathbf{r}_{\alpha}\right)+V_{\beta}\left(\mathbf{r}_{\beta}\right)+V_{\gamma}\left(\mathbf{r}_{\gamma}\right)$ is the full interaction and $E=E_{\alpha n}+q_{\alpha n}^{2} / 2 M_{\alpha}=k_{\alpha}^{2} / 2 \mu_{\alpha}+$ $q_{\alpha}^{2} / 2 M_{\alpha}$ is the total energy of the system. We split the wavefunction $\Psi_{\alpha n}^{+}$into the initial-channel wave $\Phi_{\alpha n}^{+}$ and scattered wave $\Psi_{\alpha n}^{\mathrm{sc}+}$ :

$$
\Psi_{\alpha n}^{+}\left(\mathbf{r}_{\alpha}, \boldsymbol{\rho}_{\alpha}\right)=\Phi_{\alpha n}^{+}\left(\mathbf{r}_{\alpha}, \boldsymbol{\rho}_{\alpha}\right)+\Psi_{\alpha n}^{\mathrm{sc}+}\left(\mathbf{r}_{\alpha}, \boldsymbol{\rho}_{\alpha}\right) .
$$

A slightly different scattering process may also take place within the same three-body system at the same total energy $E$, the one where in the initial channel (in the time-reversed picture this will be the final state) all three particles are in the continuum which we call $3 \rightarrow 3$ scattering. The wavefunction $\Psi_{0}^{-}$describing this process is also an eigenstate of the same Hamiltonian $H$,

$$
(E-H) \Psi_{0}^{-}\left(\mathbf{r}_{\alpha}, \boldsymbol{\rho}_{\alpha}\right)=0,
$$

but with incoming scattered-wave boundary conditions. In the total wavefunction $\Psi_{0}^{-}\left(\mathbf{r}_{\alpha}, \boldsymbol{\rho}_{\alpha}\right)$ we separate the part describing the unscattered state of three free particles, denoted $\Phi_{0}^{-}\left(\mathbf{r}_{\alpha}, \boldsymbol{\rho}_{\alpha}\right)$ and which $\Psi_{0}^{-}$is being developed to (in the absence of the Coulomb interaction this would simply be the three-body plane wave),

$$
\Psi_{0}^{-}\left(\mathbf{r}_{\alpha}, \boldsymbol{\rho}_{\alpha}\right)=\Phi_{0}^{-}\left(\mathbf{r}_{\alpha}, \boldsymbol{\rho}_{\alpha}\right)+\Psi_{0}^{\mathrm{sc}-}\left(\mathbf{r}_{\alpha}, \boldsymbol{\rho}_{\alpha}\right) .
$$


Asymptotic boundary conditions that the wavefunctions $\Psi_{\alpha n}^{+}$and $\Psi_{0}^{-}$satisfy are compiled in Ref. [12].

\subsection{Amplitudes of direct and rearrangement scattering and Coulomb breakup in prior form}

Now we use $\Psi_{\alpha n}^{+}$and $\Psi_{0}^{-}$to derive amplitudes for different scattering processes. This time we need an incomplete inner product in the six-dimensional configuration space. Such a product of two arbitrary functions $\Psi_{i}$ and $\Psi_{f}$ is written as a volume integral

$$
\left\langle\Psi_{f} \mid \Psi_{i}\right\rangle_{R_{0}}=\int_{R \leq R_{0}} d \mathbf{r}_{\alpha} d \boldsymbol{\rho}_{\alpha} \Psi_{f}^{*}\left(\mathbf{r}_{\beta}, \boldsymbol{\rho}_{\beta}\right) \Psi_{i}\left(\mathbf{r}_{\alpha}, \boldsymbol{\rho}_{\alpha}\right),(
$$

where the integration is limited to the volume of a sixdimensional hypersphere of radius $R_{0}$. A hyperradius in the six-dimensional configurations space is defined according to $R=\left(\mu_{\alpha} r_{\alpha}^{2}+M_{\alpha} \rho_{\alpha}^{2}\right)^{1 / 2}$.

Taking into account Eq. (38) we can write Eq. (37) as

$$
(E-H) \Psi_{\alpha n}^{\mathrm{sc}+}\left(\mathbf{r}_{\alpha}, \boldsymbol{\rho}_{\alpha}\right)=(H-E) \Phi_{\alpha n}^{+}\left(\mathbf{r}_{\alpha}, \boldsymbol{\rho}_{\alpha}\right) .(
$$

Let us multiply Eq. (42) by $\Psi_{0}^{-*}\left(\mathbf{r}_{\alpha}, \boldsymbol{\rho}_{\alpha}\right)$ from the left and integrate the result over the volume of a hypersphere of radius $R_{0}$ :

$$
\left\langle\Psi_{0}^{-} \mid(E-H) \Psi_{\alpha n}^{\mathrm{sc}+}\right\rangle_{R_{0}}=\left\langle\Psi_{0}^{-} \mid(H-E) \Phi_{\alpha n}^{+}\right\rangle_{R_{0}}
$$

We also have

$$
\left\langle(E-H) \Psi_{0}^{-} \mid \Psi_{\alpha n}^{\mathrm{sc}+}\right\rangle_{R_{0}}=0,
$$

which is true for any $R_{0}$ simply due to Eq. (39). Now we subtract Eq. (44) from (43) to get

$$
\begin{aligned}
\left\langle\Psi_{0}^{-} \mid(E-H) \Psi_{\alpha n}^{\mathrm{sc}+}\right\rangle_{R_{0}}- & \left\langle(E-H) \Psi_{0}^{-} \mid \Psi_{\alpha n}^{\mathrm{sc}+}\right\rangle_{R_{0}}= \\
& \left\langle\Psi_{0}^{-} \mid(H-E) \Phi_{\alpha n}^{+}\right\rangle_{R_{0}} .
\end{aligned}
$$

Despite of the fact that both $\Psi_{0}^{-}$and $\Psi_{\alpha n}^{\mathrm{sc}+}$ are non- $L^{2}$ functions, terms of the form $\left\langle\Psi_{0}^{-}|(E-V)| \Psi_{\alpha n}^{\mathrm{sc}+}\right\rangle_{R_{0}}$ are finite due to the limited space (regardless of the longrange nature of the potential). Therefore, canceling them we look at the limit of this equation as $R_{0} \rightarrow \infty$

$$
\begin{array}{r}
\lim _{R_{0} \rightarrow \infty}\left[-\left\langle\Psi_{0}^{-} \mid H_{0} \Psi_{\alpha n}^{\mathrm{sc}+}\right\rangle+\left\langle H_{0} \Psi_{0}^{-} \mid \Psi_{\alpha n}^{\mathrm{sc}+}\right\rangle\right]_{R_{0}}= \\
\lim _{R_{0} \rightarrow \infty}\left\langle\Psi_{0}^{-} \mid(H-E) \Phi_{\alpha n}^{+}\right\rangle_{R_{0}} .
\end{array}
$$

As in the two-body case, the meaning of this quantity will become clear when we evaluate the limits.

Parameter $R_{0}$ can go to infinity with the system being in $\Omega_{0}$ or $\Omega_{\alpha}, \alpha=1,2,3$. An essential feature of the term on the LHS of Eq. (46) is that it is easily transformed into an integral over the hypersurface of radius $R_{0}$ so that the result depends only on the behavior of the wavefunctions on this surface. For this integral the knowledge of the wavefunctions anywhere inside the surface is not required. Then it can be evaluated using the asymptotic forms of the wavefunctions in the corresponding asymptotic domain.

Let us start from $\Omega_{0}$ domain. If $R_{0} \rightarrow \infty$ in $\Omega_{0}$ then for the LHS of Eq. (46) we have

$$
\begin{aligned}
\text { LHS }= & \frac{1}{2\left(\mu_{\alpha} M_{\alpha}\right)^{3 / 2}} \lim _{R_{0} \rightarrow \infty} R_{0}^{5} \int d \hat{\mathbf{r}}_{\alpha} d \hat{\boldsymbol{\rho}}_{\alpha} \\
& \times \int_{0}^{\pi / 2} d \varphi_{\alpha} \sin ^{2} \varphi_{\alpha} \cos ^{2} \varphi_{\alpha} \\
& \times\left[\Psi_{0}^{-*}\left(\mathbf{r}_{\alpha}, \boldsymbol{\rho}_{\alpha}\right) \frac{\partial}{\partial R} \Psi_{\alpha n}^{\mathrm{sc}+}\left(\mathbf{r}_{\alpha}, \boldsymbol{\rho}_{\alpha}\right)\right. \\
& \left.-\Psi_{\alpha n}^{\mathrm{sc}+}\left(\mathbf{r}_{\alpha}, \boldsymbol{\rho}_{\alpha}\right) \frac{\partial}{\partial R} \Psi_{0}^{-*}\left(\mathbf{r}_{\alpha}, \boldsymbol{\rho}_{\alpha}\right)\right]_{R=R_{0}},
\end{aligned}
$$

where $\varphi_{\alpha}=\arctan \left[\left(\mu_{\alpha} / M_{\alpha}\right)^{1 / 2} r_{\alpha} / \rho_{\alpha}\right]$ is a hyperangle, $0 \leq \varphi_{\alpha} \leq \pi / 2$. Here we first transformed $H_{0}$ from $\left(\mathbf{r}_{\alpha}, \boldsymbol{\rho}_{\alpha}\right)$ into $\left(R, \widehat{\mathbf{r}}_{\alpha}, \widehat{\boldsymbol{\rho}}_{\alpha}, \varphi_{\alpha}\right)$-variables and then made use of Green's theorem to transform the volume integral into the surface integral. Now we can use the asymptotic forms for the wave functions [12] and perform differentiation to find out that this is an extremely oscillatory integral as $R_{0} \rightarrow \infty$. Therefore, only points of stationary phase in $\varphi_{\alpha}$, if there are any, should contribute to the integral. Calculating the integral by means of the stationary-phase method we arrive at

$$
\mathrm{LHS}=T\left(\mathbf{k}_{\alpha}, \mathbf{q}_{\alpha}\right)
$$

where $T\left(\mathbf{k}_{\alpha}, \mathbf{q}_{\alpha}\right)$ is the amplitude of the of the scattered wave in $\Omega_{0}$. Therefore, in $\Omega_{0}$ domain Eq. (46) is written as

$$
T\left(\mathbf{k}_{\alpha}, \mathbf{q}_{\alpha}\right)=\lim _{R_{0} \rightarrow \infty}\left\langle\Psi_{0}^{-} \mid(H-E) \Phi_{\alpha n}^{+}\right\rangle_{R_{0}} .
$$

In other words, if scattering takes place into $\Omega_{0}$ domain then expression $\lim _{R_{0} \rightarrow \infty}\left\langle\Psi_{0}^{-} \mid(H-E) \Phi_{\alpha n}^{+}\right\rangle_{R_{0}}$ represents the breakup amplitude. If after the collision the products of scattering turn out to be in $\Omega_{\alpha}$ or in $\Omega_{\beta}$ domains then we have to differentiate whether all three particles are in the continuum or just one is. If all three are in continuum then in a similar way we used for $\Omega_{0}$ we can show that $\lim _{R_{0} \rightarrow \infty}\left\langle\Psi_{0}^{-} \mid(H-E) \Phi_{\alpha n}^{+}\right\rangle_{R_{0}}$ again represents the breakup amplitude. Thus, Eq. (49) defines the breakup amplitude in all asymptotic domains.

If after the collision the products of scattering form a two-fragment channel then instead of $\Psi_{0}^{-}$we will need the total scattering wavefunction which develops into the wavefunction of this two-fragment channel. We start from $\Omega_{\alpha}$ domain which corresponds to direct scattering. In this case the total scattering wavefunction we need is $\Psi_{\alpha m}^{-}$. Let us multiply Eq. (42) by $\Psi_{\alpha m}^{-*}\left(\mathbf{r}_{\alpha}, \boldsymbol{\rho}_{\alpha}\right)$ from the left and integrate the result over the volume of a hypersphere of radius $R_{0}$ :

$$
\left\langle\Psi_{\alpha m}^{-} \mid(E-H) \Psi_{\alpha n}^{\mathrm{sc}+}\right\rangle_{R_{0}}=\left\langle\Psi_{\alpha m}^{-} \mid(H-E) \Phi_{\alpha n}^{+}\right\rangle_{R_{0}}
$$


Now we subtract $\left\langle(E-H) \Psi_{\alpha m}^{-} \mid \Psi_{\alpha n}^{\mathrm{sc}+}\right\rangle_{R_{0}}=0$ from Eq. (50) to get

$$
\begin{aligned}
-\left\langle\Psi_{\alpha m}^{-} \mid H_{0} \Psi_{\alpha n}^{\mathrm{sc}+}\right\rangle_{R_{0}} & +\left\langle H_{0} \Psi_{\alpha m}^{-} \mid \Psi_{\alpha n}^{\mathrm{sc}+}\right\rangle_{R_{0}}= \\
& \left\langle\Psi_{\alpha m}^{-} \mid(H-E) \Phi_{\alpha n}^{+}\right\rangle_{R_{0}} .
\end{aligned}
$$

We again consider the limit of this equation as $R_{0} \rightarrow$ $\infty$. Since this time $R_{0} \rightarrow \infty$ in $\Omega_{\alpha}$ then on the LHS of Eq. (51) we have

$$
\begin{aligned}
\text { LHS }= & \frac{1}{2 M_{\alpha}} \lim _{R_{0} \rightarrow \infty} R_{0}^{2} \int d \mathbf{r}_{\alpha} d \hat{\boldsymbol{\rho}}_{\alpha} \\
& \times\left[\Psi_{\alpha m}^{-*}\left(\mathbf{r}_{\alpha}, \boldsymbol{\rho}_{\alpha}\right) \frac{\partial}{\partial \rho_{\alpha}} \Psi_{\alpha n}^{\mathrm{sc}+}\left(\mathbf{r}_{\alpha}, \boldsymbol{\rho}_{\alpha}\right)\right. \\
& \left.-\Psi_{\alpha n}^{\mathrm{sc}+}\left(\mathbf{r}_{\alpha}, \boldsymbol{\rho}_{\alpha}\right) \frac{\partial}{\partial \rho_{\alpha}} \Psi_{\alpha m}^{-*}\left(\mathbf{r}_{\alpha}, \boldsymbol{\rho}_{\alpha}\right)\right]_{\rho_{\alpha}=R_{0}} .
\end{aligned}
$$

Here we transformed only one of the volume integrals into the surface integral (the wave functions fall off exponentially in the other two-body subspace). Using the asymptotic forms of the wavefunctions [12] and the orthogonality of the the two-particle bound state wavefunctions and calculating the integral we arrive at

$$
\mathrm{LHS}=F\left(\mathbf{q}_{\alpha m}, \mathbf{q}_{\alpha n}\right)
$$

where $F\left(\mathbf{q}_{\alpha m}, \mathbf{q}_{\alpha n}\right)$ is the amplitude of the wave scattered into channel $\alpha$. Thus as $R_{0} \rightarrow \infty$ Eq. (51) is in fact written as

$$
F\left(\mathbf{q}_{\alpha m}, \mathbf{q}_{\alpha n}\right)=\lim _{R_{0} \rightarrow \infty}\left\langle\Psi_{\alpha m}^{-} \mid(H-E) \Phi_{\alpha n}^{+}\right\rangle_{R_{0}} .
$$

In other words we have got a definition for the direct scattering (elastic and excitation) amplitude. Finally, taking $R_{0} \rightarrow \infty$ in $\Omega_{\beta}$ (i.e., the final state belongs to channel $\beta$ ) and calculating the limit of Eq. (51) we get a definition for the amplitude of the rearrangement scattering

$$
G\left(\mathbf{q}_{\beta m}, \mathbf{q}_{\alpha n}\right)=\lim _{R_{0} \rightarrow \infty}\left\langle\Psi_{\beta m}^{-} \mid(H-E) \Phi_{\alpha n}^{+}\right\rangle_{R_{0}} .
$$

\subsection{Scattering and breakup amplitudes in post form}

Taking into account Eq. (40) we can write Eq. (39) as

$$
(E-H) \Psi_{0}^{\mathrm{sc}-}\left(\mathbf{r}_{\alpha}, \boldsymbol{\rho}_{\alpha}\right)=(H-E) \Phi_{0}^{-}\left(\mathbf{r}_{\alpha}, \boldsymbol{\rho}_{\alpha}\right) .
$$

Let us take the complex conjugate of Eq. (56) and multiply it by $\Psi_{\alpha n}^{+}\left(\mathbf{r}_{\alpha}, \boldsymbol{\rho}_{\alpha}\right)$ from right. Then integrating the result over the volume of a hypersphere of radius $R_{0}$ we get

$$
\left\langle(E-H) \Psi_{0}^{\mathrm{sc}-} \mid \Psi_{\alpha n}^{+}\right\rangle_{R_{0}}=\left\langle(E-H) \Phi_{0}^{-} \mid \Psi_{\alpha n}^{+}\right\rangle_{R_{0}} .
$$

We also consider

$$
\left\langle\Psi_{0}^{\mathrm{sc}-} \mid(E-H) \Psi_{\alpha n}^{+}\right\rangle_{R_{0}}=0
$$

which is again valid for any $R_{0}$ due to Eq. (37). Now we subtract Eq. (58) from (57)

$$
\begin{aligned}
\left\langle(E-H) \Psi_{0}^{\mathrm{sc}-} \mid \Psi_{\alpha n}^{+}\right\rangle_{R_{0}} & -\left\langle\Psi_{0}^{\mathrm{sc}-} \mid(E-H) \Psi_{\alpha n}^{+}\right\rangle_{R_{0}} \\
= & \left\langle(E-H) \Phi_{0}^{-} \mid \Psi_{\alpha n}^{+}\right\rangle_{R_{0}},
\end{aligned}
$$

which reduces to

$$
\begin{array}{r}
-\left\langle H_{0} \Psi_{0}^{\mathrm{sc}-} \mid \Psi_{\alpha n}^{+}\right\rangle_{R_{0}}+\left\langle\Psi_{0}^{\mathrm{sc}-} \mid H_{0} \Psi_{\alpha n}^{+}\right\rangle_{R_{0}}= \\
\left.\left(\left\langle\Phi_{0}^{-}\right|(H-E)\right) \Psi_{\alpha n}^{+}\right\rangle_{R_{0}} .
\end{array}
$$

Calculations of the limit of the LHS of Eq. (60) are similar to those of Eq. (46) leading to Eqs. (49), (54) and (55). Depending on whether the $R_{0} \rightarrow \infty$ limit is taken in domains $\Omega_{0}, \Omega_{\alpha}$ or $\Omega_{\beta}$ we have

$$
\begin{aligned}
T\left(\mathbf{k}_{\alpha}, \mathbf{q}_{\alpha}\right) & =\lim _{R_{0} \rightarrow \infty}\left\langle(E-H) \Phi_{0}^{-} \mid \Psi_{\alpha n}^{+}\right\rangle_{R_{0}}, \\
F\left(\mathbf{q}_{\alpha m}, \mathbf{q}_{\alpha n}\right) & =\lim _{R_{0} \rightarrow \infty}\left\langle(E-H) \Phi_{\alpha m}^{-} \mid \Psi_{\alpha n}^{+}\right\rangle_{R_{0}}, \\
G\left(\mathbf{q}_{\beta m}, \mathbf{q}_{\alpha n}\right) & =\lim _{R_{0} \rightarrow \infty}\left\langle(E-H) \Phi_{\beta m}^{-} \mid \Psi_{\alpha n}^{+}\right\rangle_{R_{0}},
\end{aligned}
$$

respectively. Thus we get alternative representations for the breakup, scattering and rearrangement amplitudes. These are the post forms of the amplitudes. In particular, the definition given in Eq. (61) resolves the problem of the post form of the Coulomb breakup amplitude mentioned earlier.

\subsection{Conventional forms of the scattering and breakup amplitudes}

As it will be shown in the following subsection the prior and post forms of the breakup amplitude can take surface-integral forms (see Eqs. (81) and (83)). These surface integrals are similar in form to those calculated in Sec. 3.1 and 3.2. They can be calculated using the same analysis and shown to yield the same result, i.e. the breakup amplitude. Therefore Eq. (46) and the limit of (60) as $R_{0} \rightarrow \infty$ are identities and that both sides of these equations represent the breakup amplitude. The same is true for the surface-integral representations of the direct scattering and rearrangement amplitudes. Therefore, Eqs. (49), (54) and (55) can in fact be written as

$$
\begin{aligned}
& T^{\operatorname{pr}}\left(\mathbf{k}_{\alpha}, \mathbf{q}_{\alpha}\right)=\left\langle\Psi_{0}^{-} \mid(H-E) \Phi_{\alpha n}^{+}\right\rangle, \\
& F^{\operatorname{pr}}\left(\mathbf{q}_{\alpha m}, \mathbf{q}_{\alpha n}\right)=\left\langle\Psi_{\alpha m}^{-} \mid(H-E) \Phi_{\alpha n}^{+}\right\rangle, \\
& G^{\operatorname{pr}}\left(\mathbf{q}_{\beta m}, \mathbf{q}_{\alpha n}\right)=\left\langle\Psi_{\beta m}^{-} \mid(H-E) \Phi_{\alpha n}^{+}\right\rangle
\end{aligned}
$$

and Eqs. (61)-(63) as

$$
\begin{aligned}
& T^{\mathrm{pt}}\left(\mathbf{k}_{\alpha}, \mathbf{q}_{\alpha}\right)=\left\langle(E-H) \Phi_{0}^{-} \mid \Psi_{\alpha n}^{+}\right\rangle, \\
& F^{\mathrm{pt}}\left(\mathbf{q}_{\alpha m}, \mathbf{q}_{\alpha n}\right)=\left\langle(E-H) \Phi_{\alpha m}^{-} \mid \Psi_{\alpha n}^{+}\right\rangle, \\
& G^{\mathrm{pt}}\left(\mathbf{q}_{\beta m}, \mathbf{q}_{\alpha n}\right)=\left\langle(E-H) \Phi_{\beta m}^{-} \mid \Psi_{\alpha n}^{+}\right\rangle .
\end{aligned}
$$


We note that when the interactions between all three pairs are short ranged then

$$
\Phi_{\alpha n}^{ \pm}\left(\mathbf{r}_{\alpha}, \boldsymbol{\rho}_{\alpha}\right) \rightarrow e^{i \mathbf{q}_{\alpha n} \cdot \boldsymbol{\rho}_{\alpha}} \phi_{\alpha n}\left(\mathbf{r}_{\alpha}\right) .
$$

This state satisfies the equation

$$
(H-E) e^{i \mathbf{q}_{\alpha n} \cdot \boldsymbol{\rho}_{\alpha}} \phi_{\alpha n}\left(\mathbf{r}_{\alpha}\right)=\bar{V}_{\alpha} e^{i \mathbf{q}_{\alpha n} \cdot \boldsymbol{\rho}_{\alpha}} \phi_{\alpha n}\left(\mathbf{r}_{\alpha}\right),
$$

where $\bar{V}_{\alpha}=V-V_{\alpha}$. At the same time if we have 3 particles in the final channel then

$$
\Phi_{0}^{-}\left(\mathbf{r}_{\alpha}, \boldsymbol{\rho}_{\alpha}\right) \rightarrow e^{i \mathbf{k}_{\alpha} \cdot \mathbf{r}_{\alpha}+i \mathbf{q}_{\alpha} \cdot \boldsymbol{\rho}_{\alpha}},
$$

for which we have

$$
(H-E) e^{i \mathbf{k}_{\alpha} \cdot \mathbf{r}_{\alpha}+i \mathbf{q}_{\alpha} \cdot \boldsymbol{\rho}_{\alpha}}=V e^{i \mathbf{k}_{\alpha} \cdot \mathbf{r}_{\alpha}+i \mathbf{q}_{\alpha} \cdot \boldsymbol{\rho}_{\alpha}} .
$$

Therefore, the new generalized forms of the amplitudes (64)-(69) reduce to the standard definitions

$$
\begin{aligned}
& T^{\operatorname{pr}}\left(\mathbf{k}_{\alpha}, \mathbf{q}_{\alpha}\right)=\left\langle\Psi_{0}^{-}\left|\bar{V}_{\alpha}\right| \phi_{\alpha n}, \mathbf{q}_{\alpha n}\right\rangle, \\
& F^{\operatorname{pr}}\left(\mathbf{q}_{\alpha m}, \mathbf{q}_{\alpha n}\right)=\left\langle\Psi_{\alpha m}^{-}\left|\bar{V}_{\alpha}\right| \phi_{\alpha n}, \mathbf{q}_{\alpha n}\right\rangle, \\
& G^{\mathrm{pr}}\left(\mathbf{q}_{\beta m}, \mathbf{q}_{\alpha n}\right)=\left\langle\Psi_{\beta m}^{-}\left|\bar{V}_{\alpha}\right| \phi_{\alpha n}, \mathbf{q}_{\alpha n}\right\rangle
\end{aligned}
$$

and

$$
\begin{aligned}
& T^{\mathrm{pt}}\left(\mathbf{k}_{\alpha}, \mathbf{q}_{\alpha}\right)=\left\langle\mathbf{q}_{\alpha}, \mathbf{k}_{\alpha}|V| \Psi_{\alpha n}^{+}\right\rangle, \\
& F^{\mathrm{pt}}\left(\mathbf{q}_{\alpha m}, \mathbf{q}_{\alpha n}\right)=\left\langle\mathbf{q}_{\alpha m}, \phi_{\alpha m}\left|\bar{V}_{\alpha}\right| \Psi_{\alpha n}^{+}\right\rangle, \\
& G^{\mathrm{pt}}\left(\mathbf{q}_{\beta m}, \mathbf{q}_{\alpha n}\right)=\left\langle\mathbf{q}_{\beta m}, \phi_{\beta m}\left|\bar{V}_{\beta}\right| \Psi_{\alpha n}^{+}\right\rangle .
\end{aligned}
$$

When the interactions have the Coulomb tail Eqs. (71) and (73) are not satisfied. For this reason the standard definitions (74)-(79) become invalid.

\subsection{Surface-integral forms for the scattering and breakup amplitudes}

As mentioned in the preceeding subsection both prior and post forms of the scattering and breakup amplitudes take surface integral forms. The post form of the breakup amplitude $T^{\mathrm{pt}}\left(\mathbf{k}_{\alpha}, \mathbf{q}_{\alpha}\right)$ is given by Eq. (61). Using Eq. (37) which is valid for any $R_{0}$ we can write this as

$$
\begin{aligned}
T^{\mathrm{pt}}\left(\mathbf{k}_{\alpha}, \mathbf{q}_{\alpha}\right)=\lim _{R_{0} \rightarrow \infty}[ & \left\langle H_{0} \Phi_{0}^{-} \mid \Psi_{\alpha n}^{+}\right\rangle \\
& \left.-\left\langle\Phi_{0}^{-} \mid H_{0} \Psi_{\alpha n}^{+}\right\rangle\right]_{R_{0}} .
\end{aligned}
$$

Transforming the volume integral into a surface integral we get

$$
\begin{aligned}
T^{\mathrm{pt}}\left(\mathbf{k}_{\alpha}, \mathbf{q}_{\alpha}\right)= & -\frac{1}{2\left(\mu_{\alpha} M_{\alpha}\right)^{3 / 2}} \lim _{R_{0} \rightarrow \infty} R_{0}^{5} \int d \hat{\mathbf{r}}_{\alpha} d \hat{\boldsymbol{\rho}}_{\alpha} \\
& \times \int_{0}^{\pi / 2} d \varphi_{\alpha} \sin ^{2} \varphi_{\alpha} \cos ^{2} \varphi_{\alpha} \\
& \times\left[\Psi_{\alpha n}^{+}\left(\mathbf{r}_{\alpha}, \boldsymbol{\rho}_{\alpha}\right) \frac{\partial}{\partial R} \Phi_{0}^{-*}\left(\mathbf{r}_{\alpha}, \boldsymbol{\rho}_{\alpha}\right)\right. \\
& \left.-\Phi_{0}^{-*}\left(\mathbf{r}_{\alpha}, \boldsymbol{\rho}_{\alpha}\right) \frac{\partial}{\partial R} \Psi_{\alpha n}^{+}\left(\mathbf{r}_{\alpha}, \boldsymbol{\rho}_{\alpha}\right)\right]_{R=R_{0}}
\end{aligned}
$$

Thus the breakup amplitude in the post form is written as a five-dimensional surface integral. In the prior form of this amplitude and also both in the post and prior forms of scattering and rearrangement amplitudes only one of the three-dimensional volume integrals can be transformed into a surface integral. Consider, for instance, the prior form of the breakup amplitude. Using Eq. (39) we get from Eq. (49)

$$
\begin{aligned}
T^{\mathrm{pr}}\left(\mathbf{k}_{\alpha}, \mathbf{q}_{\alpha}\right)=\lim _{R_{0} \rightarrow \infty}[ & \left\langle\Psi_{0}^{-} \mid H_{0} \Phi_{\alpha n}^{+}\right\rangle \\
& \left.-\left\langle H_{0} \Psi_{0}^{-} \mid \Phi_{\alpha n}^{+}\right\rangle\right]_{R_{0}} .
\end{aligned}
$$

This can be written as

$$
\begin{aligned}
T^{\mathrm{pr}}\left(\mathbf{k}_{\alpha}, \mathbf{q}_{\alpha}\right)= & -\frac{1}{2 M_{\alpha}} \lim _{R_{0} \rightarrow \infty} R_{0}^{2} \int d \mathbf{r}_{\alpha} d \hat{\boldsymbol{\rho}}_{\alpha} \\
& \times\left[\Psi_{0}^{-*}\left(\mathbf{r}_{\alpha}, \boldsymbol{\rho}_{\alpha}\right) \frac{\partial}{\partial \rho_{\alpha}} \Phi_{\alpha n}^{+}\left(\mathbf{r}_{\alpha}, \boldsymbol{\rho}_{\alpha}\right)\right. \\
& -\Phi_{\alpha n}^{+}\left(\mathbf{r}_{\alpha}, \boldsymbol{\rho}_{\alpha}\right) \\
& \left.\times \frac{\partial}{\partial \rho_{\alpha}} \Psi_{0}^{-*}\left(\mathbf{r}_{\alpha}, \boldsymbol{\rho}_{\alpha}\right)\right]_{\rho_{\alpha}=R_{0}}
\end{aligned}
$$

Note that above we took into account the fact that the surface integral in the other two-body space is zero as $r_{\alpha} \rightarrow \infty$ (the function $\Phi_{\alpha n}^{+}$falls off exponentially in this variable). Similarly we get

$$
\begin{aligned}
F^{\mathrm{pr}}\left(\mathbf{q}_{\alpha m}, \mathbf{q}_{\alpha n}\right)= & -\frac{1}{2 M_{\alpha}} \lim _{R_{0} \rightarrow \infty} R_{0}^{2} \int d \mathbf{r}_{\alpha} d \hat{\boldsymbol{\rho}}_{\alpha} \\
& \times\left[\Psi_{\alpha m}^{-*}\left(\mathbf{r}_{\alpha}, \boldsymbol{\rho}_{\alpha}\right) \frac{\partial}{\partial \rho_{\alpha}} \Phi_{\alpha n}^{+}\left(\mathbf{r}_{\alpha}, \boldsymbol{\rho}_{\alpha}\right)\right. \\
& -\Phi_{\alpha n}^{+}\left(\mathbf{r}_{\alpha}, \boldsymbol{\rho}_{\alpha}\right) \\
& \left.\times \frac{\partial}{\partial \rho_{\alpha}} \Psi_{\alpha m}^{-*}\left(\mathbf{r}_{\alpha}, \boldsymbol{\rho}_{\alpha}\right)\right]_{\rho_{\alpha}=R_{0}}, \quad(84) \\
G^{\mathrm{pr}}\left(\mathbf{q}_{\beta m}, \mathbf{q}_{\alpha n}\right)= & -\frac{1}{2 M_{\alpha}} \lim _{0} \rightarrow R_{0}^{2} \int d \mathbf{r}_{\alpha} d \hat{\boldsymbol{\rho}}_{\alpha} \\
& \times\left[\Psi_{\beta m}^{-*}\left(\mathbf{r}_{\alpha}, \boldsymbol{\rho}_{\alpha}\right) \frac{\partial}{\partial \rho_{\alpha}} \Phi_{\alpha n}^{+}\left(\mathbf{r}_{\alpha}, \boldsymbol{\rho}_{\alpha}\right)\right. \\
& -\Phi_{\alpha n}^{+}\left(\mathbf{r}_{\alpha}, \boldsymbol{\rho}_{\alpha}\right) \\
& \left.\times \frac{\partial}{\partial \rho_{\alpha}} \Psi_{\beta m}^{-*}\left(\mathbf{r}_{\alpha}, \boldsymbol{\rho}_{\alpha}\right)\right]_{\rho_{\alpha}=R_{0}}, \quad(85)
\end{aligned}
$$

and in post forms

$$
\begin{aligned}
F^{\mathrm{pt}}\left(\mathbf{q}_{\alpha m}, \mathbf{q}_{\alpha n}\right)= & -\frac{1}{2 M_{\alpha}} \lim _{R_{0} \rightarrow \infty} R_{0}^{2} \int d \mathbf{r}_{\alpha} d \hat{\boldsymbol{\rho}}_{\alpha} \\
& \times\left[\Psi_{\alpha n}^{+}\left(\mathbf{r}_{\alpha}, \boldsymbol{\rho}_{\alpha}\right) \frac{\partial}{\partial \rho_{\alpha}} \Phi_{\alpha m}^{-*}\left(\mathbf{r}_{\alpha}, \boldsymbol{\rho}_{\alpha}\right)\right. \\
& -\Phi_{\alpha m}^{-*}\left(\mathbf{r}_{\alpha}, \boldsymbol{\rho}_{\alpha}\right) \\
& \left.\times \frac{\partial}{\partial \rho_{\alpha}} \Psi_{\alpha n}^{+}\left(\mathbf{r}_{\alpha}, \boldsymbol{\rho}_{\alpha}\right)\right]_{\rho_{\alpha}=R_{0}}, \quad(86) \\
G^{\mathrm{pt}}\left(\mathbf{q}_{\beta m}, \mathbf{q}_{\alpha n}\right)= & -\frac{1}{2 M_{\beta}} \lim _{R_{0} \rightarrow \infty} R_{0}^{2} \int d \mathbf{r}_{\beta} d \hat{\boldsymbol{\rho}}_{\beta}
\end{aligned}
$$


$19^{\text {th }}$ International IUPAP Conference on Few-Body Problems in Physics

$$
\begin{aligned}
& \times\left[\Psi_{\alpha n}^{+}\left(\mathbf{r}_{\beta}, \boldsymbol{\rho}_{\beta}\right) \frac{\partial}{\partial \rho_{\beta}} \Phi_{\beta m}^{-*}\left(\mathbf{r}_{\beta}, \boldsymbol{\rho}_{\beta}\right)\right. \\
& -\Phi_{\beta m}^{-*}\left(\mathbf{r}_{\beta}, \boldsymbol{\rho}_{\beta}\right) \\
& \left.\times \frac{\partial}{\partial \rho_{\beta}} \Psi_{\alpha n}^{+}\left(\mathbf{r}_{\beta}, \boldsymbol{\rho}_{\beta}\right)\right]_{\rho_{\alpha}=R_{0}}
\end{aligned}
$$

What is the advantage of the surface-integral representations over the volume-integral forms? As we can see from the surface-integral forms for the amplitudes, they are ideal for practical calculations in partial waves as integrals over solid angles are taken [9]. Moreover, the corresponding results depend only on the behavior of the wavefunctions on the hypersurface of radius $R_{0}$, meaning that for these integrals the knowledge of the wavefunctions anywhere inside the surface is not required. Therefore, they can be evaluated using the asymptotic forms of the wavefunctions in the corresponding asymptotic domain. The main advantage of the surface forms is that the asymptotic channel wavefunctions $\Phi_{0}^{-}, \Phi_{\alpha m}^{ \pm}$and $\Phi_{\beta m}^{-}$necessary for extracting the breakup, scattering and rearrangement amplitudes do not have to be exact. They can be replaced by other suitable functions making sure that magnitudes of the amplitudes are still calculated exactly, see Ref. [12] for more discussion.

\section{Discussion}

In order to solve a scattering problem, first, one has to find the total wavefunction describing the scattering process. The second part consists in the extraction of the necessary scattering amplitudes from this wave function for the purpose of calculating the cross sections. So far we have resolved problems of the theory related to extracting the information about the scattering event. When the total scattering wavefunction is available the scattering amplitudes can be reliably extracted from it using the new definitions regardless the long-range nature of the interactions. Once the amplitudes are available calculations of corresponding cross sections are straightforward (see, e.g., [15]). As mentioned earlier, there are sophisticated numerical methods which can provide reliable numerical solution to the Schrödinger equation in some special cases. However, in case of three distinguishable particles where rearrangement is possible the Schrödinger equation cannot provide a unique answer. This is because of the fact that it is impossible to specify all asymptotic boundary conditions using one set of the Jacobi variables. To overcome this difficulty Faddeev suggested [2] a set of equations which incorporates all the required asymptotic boundary conditions. However, as mentioned earlier in case of charged particles the Faddeev equations become noncompact. In other words they cannot be solved using standard numerical techniques though noncompactness generally does not exclude existence of analytic solutions. At the same time our results show that problems with the
Faddeev equations are more serious than noncompactness. Equivalent sets of the Faddeev equations can be written for the wavefunction, resolvent of the Green's function or T-matrix. Let us consider the equations for the components of the three-body T-matrix. The starting point for these equations are old (conventional) definitions in terms of the interaction potentials which are simply not correct for charged particles. This implies that any results derived from old definitions are valid strictly for short-range potentials. For the Coulombic potentials they might be simply incorrect. Thus, the Faddeev equations in the presence of long-range Coulomb interactions require careful inspection. It may be possible to formulate the Faddeev equations in a form that would not require screening and renormalization along the lines of the present surface-integral formalism.

The main ingredient of the Faddeev equations is the off-the-energy-shell two-body T-matrix. For charged particles it is the off-shell Coulomb two-body T-matrix. The theory of the Coulomb T-matrix, which started from pioneering work of Schwinger in the 1940's (fist published in Ref. [16]), is believed to be well developed and complete [1]. However, as we mentioned earlier the off-shell Coulomb T-matrix has no on-shell limit. It diverges as the on-shell point is approached where physical observables are extracted. Therefore, it cannot be directly used to calculate the physical Coulomb scattering amplitude. In particular, it appears obvious that if used it can only exacerbate the problems of the Faddeev equations. The theory is based on the conception that the Coulomb T-matrix cannot be defined directly on the energy shell. However, in the present work we have shown this to be a misconception and introduced the onshell Coulomb T-matrix which directly gives the physical scattering amplitude. In other words, the on-shell Coulomb T-matrix presented here has no singularities and therefore does not require renormalisation. This means that the conventional theory of the Coulomb Tmatrix must be abandoned altogether and a new theory be developed. We believe a new off-shell Coulomb T-matrix should be introduced as an analytic extension into the complex-energy plane of the on-shell $\mathrm{T}$ matrix presented in this work.

We started this paper with a general comment about divergence problems in different branches of physics, associated with fields creating $1 / r$ potentials. These annoying difficulties are consequences of imperfections in the existing theories. Renormalization is used to deal with such problems in quantum electrodynamics (QED). QED has experienced extraordinary success since Feynman, Schwinger and Tomonaga suggested the renormalization method. However, certain dissatisfaction with this theory remains [17-19]. Berestetskii, Lifshitz and Pitaevskii [20] start their well-known textbook emphasizing that "there is as yet no logically consistent and complete relativistic quantum theory. ... The lack of complete logical consistency in this theory is shown by the occurrence of 
divergent expressions when the mathematical formalism is directly applied, although there are quite welldefined ways of eliminating these divergences. Nevertheless, such methods remain, to a considerable extent, semiempirical rules, and our confidence in the correctness of the results is ultimately based only on their excellent agreement with experiment, not on the internal consistency or logical ordering of the fundamental principles of the theory." While another popular author Ryder [21] concludes that "despite the comparative success of renormalisation theory the feeling remains that there ought to be a more satisfactory way of doing things." Unfortunately, these concerns are largely ignored by the scientific community. We believe there must be a logically consistent solution to these problems where the mathematical formalism is directly applicable without the need for renormalization. In our case, utilising a surface-integral approach and focusing on integrated properties removes screening and renormalisation requirements in scattering theory. We suggest that a similar integrated approach may help eliminate renormalisation requirements in QED and other fields. This looks feasible at least in case of the infrared divergencies.

\section{Conclusion}

We have given a brief account of a formalism of scattering theory which is applicable to two-body and three-body systems with both short-range and Coulombic long-range potentials. The formalism is based on a surface-integral approach. New definitions for the potential scattering amplitude valid for arbitrary interactions are presented. For the Coulombic potentials these generalized definitions of the amplitude give the physical on-shell amplitude without recourse to a renormalization procedure. New prior and post forms of the breakup amplitude for a three-body system are given that are valid for both short-range and Coulombic potentials. This resolves a problem of the missing conventional post form of the Coulomb breakup amplitude. An essential feature of the surfaceintegral formulation is that it avoids any reference to the Green's functions and formal solutions of the Schrödinger equation in integral forms. Therefore, for the purpose of defining the scattering amplitudes the knowledge of a complicated analytic structure of the Green's function in the complex-energy plane is not required. This constitutes a simpler yet more general alternative to formulations adopted in textbooks on scattering theory.

This work was supported by the Australian Research Council and the U.S. Department of Energy under Grant No. DE-FG02-93ER40773.

\section{References}

1. H. van Haeringen, Charged-particle Interactions (Coulomb Press Leyden, Leiden, 1985).

2. L. D. Faddeev, Sov. Phys. -JETP 12, 1014 (1961).

3. L. D. Faddeev, Mathematical Aspects of the ThreeBody Problem in the Quantum Scattering (Israel Program for Scientific Translations, Jerusalem, 1965).

4. E. O. Alt, W. Sandhas, and H. Ziegelmann, Phys. Rev. C 17, 1981 (1978).

5. T. N. Rescigno, M. Baertschy, W. A. Isaacs, and C. W. McCurdy, Science 286, 2474 (1999).

6. M. Baertschy, T. N. Rescigno, and C. W. McCurdy, Phys. Rev. A 64, 022709 (2001).

7. I. Bray and A. T. Stelbovics, Phys. Rev. Lett. 69, 53 (1992).

8. A. S. Kadyrov, A. M. Mukhamedzhanov, A. T. Stelbovics, and I. Bray, Phys. Rev. Lett. 91, 253202 (2003).

9. A. S. Kadyrov, A. M. Mukhamedzhanov, A. T. Stelbovics, and I. Bray, Phys. Rev. A 70, 062703 (2004).

10. R. K. Peterkop, Opt. Spectrosc. 13, 87 (1962).

11. A. S. Kadyrov, I. Bray, A. M. Mukhamedzhanov, and A. T. Stelbovics, Phys. Rev. Lett. 101, 230405 (2008).

12. A. S. Kadyrov, I. Bray, A. M. Mukhamedzhanov, and A. T. Stelbovics, Ann. Phys. 324, 1516 (2009).

13. A. Messiah, Quantum Mechanics, vol. 2 (NorthHolland Publishing, Amsterdam, 1965).

14. A. S. Kadyrov, I. Bray, A. M. Mukhamedzhanov, and A. T. Stelbovics, Phys. Rev. A 72, 032712 (2005).

15. R. G. Newton, Ann. Phys. 74, 324 (1972).

16. J. Schwinger, J. Math. Phys. 5, 1606 (1964).

17. P. A. M. Dirac, in AIP Conf. Proc. 74, edited by D. W. Duke and J. F. Owens (AIP, New York, 1981), pp. 129-130.

18. W. Pauli, Nobel Lectures, Physics 1942-1962 (Elsevier, Amsterdam, 1964), pp. 27-43.

19. R. P. Feynman, QED, The Strange Theory of Light and Matter (Princeton University Press, Princeton, 1985), pp. 128-129.

20. V. B. Berestetskii, E. M. Lifshitz, and L. P. Pitaevskii, Quantum electrodynamics (Pergamon press, 1982), pp. 3-4, 2nd ed.

21. L. H. Ryder, Quantum Field Theory (Cambridge University Press, Cambridge, 1996), p. 390, 2nd ed. 